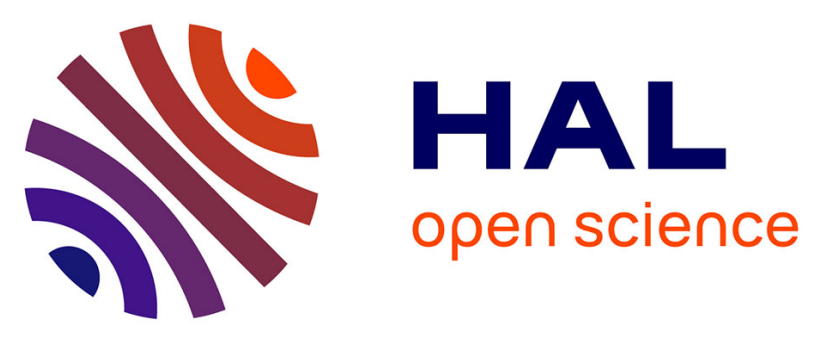

\title{
Identifying Conflicts Through Emails by Using an Emotion Ontology
}

\author{
Chahnaz Zakaria, Olivier Curé, Kamel Smaïli
}

\section{To cite this version:}

Chahnaz Zakaria, Olivier Curé, Kamel Smaïli. Identifying Conflicts Through Emails by Using an Emotion Ontology. 6th International Workshop on Natural Language Processing and Cognitive Science Collocated with 11th International Conference on Enterprise Information Systems (ICEIS 2009), May 2009, Milano, Italy. hal-01261615

\section{HAL Id: hal-01261615 https://hal.science/hal-01261615}

Submitted on 25 Jan 2016

HAL is a multi-disciplinary open access archive for the deposit and dissemination of scientific research documents, whether they are published or not. The documents may come from teaching and research institutions in France or abroad, or from public or private research centers.
L'archive ouverte pluridisciplinaire HAL, est destinée au dépôt et à la diffusion de documents scientifiques de niveau recherche, publiés ou non, émanant des établissements d'enseignement et de recherche français ou étrangers, des laboratoires publics ou privés. 


\title{
Identifying Conflicts Through Emails by Using an Emotion Ontology
}

\author{
Chahnez Zakaria ${ }^{1}$, Olivier Curé ${ }^{1}$, and Kamel Smaïli ${ }^{3}$ \\ 1 Université Paris Est, Terre Digitale IGM LabInfo \\ 5, bd Descartes Champs sur Marne, 77454 Marne la Vallée France \\ \{chahnez.zakaria, olivier.cure\}@univ-mlv.fr \\ ${ }^{2}$ Laboratory Loria, Campus Scientifique, \\ BP 23954506 Vandoeuvre Lès-Nancy \\ smailieloria.fr
}

\begin{abstract}
In the logic of text classification, this paper presents an approach to detect emails conflict exchanged between colleagues, who belong to a geographically distributed enterprise. The idea is to inform a team leader of such situation, hence to help him in preventing serious disagreement between team members. This approach uses the vector space model with TF*IDF weight to represent email; and a domain ontology of relational conflicts to determine its categories. Our study also addresses the issue of building ontology, which is made up of two phases. First we conceptualize the domain by hand, then we enrich it by using the triggers model that enables to find out terms in corpora which correspond to different conflicts.
\end{abstract}

\section{Introduction}

Geographically distributed teams of a given enterprise can overcome the problems of distance by using Computer Supported Cooperative Work (CSCW) tools. However it is still difficult for a team leader to remotely manage the emotions of its members and the conflicts that may arise between them. Such situations can complicate communication and cooperation between them. Indeed it has been proven over several decades by Elton Mayo at the Hawthorne experiments [1], that good horizontal and / or vertical relationships, in a professional environment, have a major influence on overall satisfaction provided by the work and personal productivity.

The constitution of virtual teams has accentuated the difficulty of the understanding an employee's behaviour. Nevertheless, the team leader can overcome this situation with the data generated by the CSCW tools, especially through the analysis of emails which allow to generate important textual corpora due to its large exploitation in professional environments [15]. The idea is to detect automatically, conflicts between team members through exchange of emails, so that the team leader can understand their behaviour, intervene and manage conflicts before they lead to irreversible situations. Our approach solves the task of conflict detection by classifying emails, according to our domain ontology of relational conflicts.

The remainder of the paper is organized as follows. Section 2 discusses related work. Section 3 describes our conceptualization approach of the conflicts domain in 
two stages. Section 4 describes the model which we have developed for classifying email based on the concepts of our ontology. Section 5 shows experimental results. Finally, Section 6 concludes with a discussion of future directions.

\section{Related Work}

Within the sphere of emails classification, some work deals with binary classification, as in information filtering, e.g. separating spam from good emails, other work deals with multiclass classification or classifying an email into one of many categories, e.g. routing email to the concerned service, in a company. Our study can be seen as a binary classification, because it filters emails with conflict situations, but our approach is also concerned with the multiclass classification as we also detect the type of conflict assigned to an email and provide a degree of importance.

In general, a classification model consists of two tasks: modeling the document using a model of representation, as the vector model [13], and his assignment to the topic that concerns through a classifier, as Naïve Bayes [7], Support Vector Machine (SVM) [2], etc.

Several approaches are proposed for building ontologies from corpora. They can be grouped into two categories: structural approaches based on the use of formal grammar; non-structural approaches, such as statistical approaches which must use enough corpora, in order to have reliable measures and find out interesting relationships between terms [9]. The acquisition of terms based on statistical approach exists since several decades: Enguehard and Pantera (1995) [6], Dias (2002) [5], etc. This work is based on the idea that words of the same area tend to often occur together. Similarity measures are used to identify recurrent associations of terms. The correlated terms recurrences are extracted by using different kind of measures [14]: Mutual Information, Dice coefficient, etc.

\section{Building Ontology}

The ontology technology was born as a response to the need for representation of knowledge in information systems. It allows to access not only to the terms used by the human being, but also to meaning associated to the various terms. For instance T. Berners-Lee [12] considers the ontology as a way to enable Web pages to integrate a representation of the knowledge they contain and to represent semantic links with other documents. One widely cited definition of an ontology is Gruber's [8] "an ontology is a formal, explicit, specification of a shared conceptualization". In other words, an ontology is designed to specify concepts and relations, and to make them understandable and usable by several agents (human or software).

The approach we present builds an ontology in two steps; the first consists in conceptualizing the domain of relational conflicts, based on human expertise; the second enriches it automatically by using a trigger-based model that enables to find terms in corpora which correspond to different conflicts. 


\subsection{Construction of a first conflict ontology draft}

To the best of our knowledge, there is no ontology of conflicts, that is why we decided to focus our work on emotions. In fact, conflicts could be detected through the expression of emotions. But, we are interested just by negative emotions which are at the origin of disagreement situations.

To conceptualize the conflict domain, we based our work on the classification of Antonio R Damasio [3] and Michelle Larivey [10]. We used their vocabularies, but we changed the separation criteria of emotions. The first criterion separates emotions according to the degree of conflict, the first category represents emotions that can produce substantial conflicts as disgust and hatred, the second one leads to anticipate some indirect conflicts as indifference. The second criterion deals with making the difference between personal and social emotions or distinguish social emotions from others. This is due to the fact that it is very difficult to determine a personal emotion, for instance, the sadness emotion may be social when this feeling is due to the behaviour of another colleague or friend, and may be personal when the person did not succeed to reach an objective; however, jealousy can easily be classified as social emotions. Figure 1 shows an example of the emotions conceptualized, we structure the terms of the concept into three types:

- synonymous: it regroups the synonyms of the term representing the concept

- forms: it regroups terms that indicate the expression of emotion

- causes: it regroups the reasons which may justify the expression of emotion

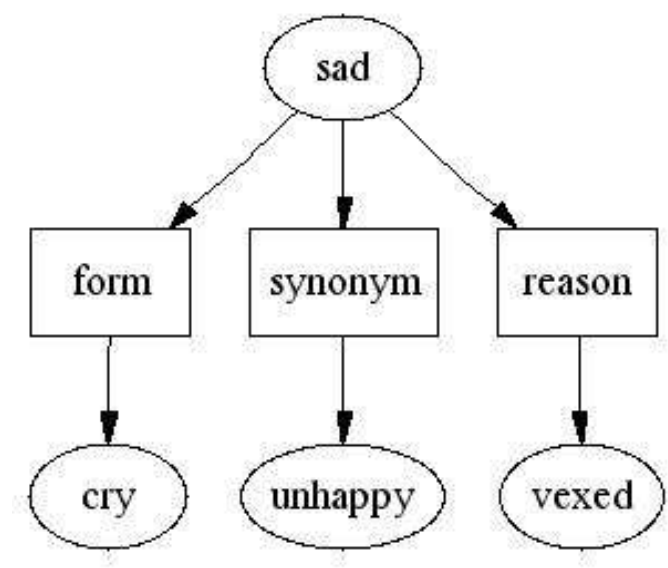

Fig. 1. The sad emotion

In the next section we present the statistical model that we used to enrich our ontology from corpora. 


\subsection{Triggers to Enrich Ontology}

Development of statistical language models is historically related to the construction of the first significant linguistic corpora [4]. For these models, a corpus represents a raw material, it is used to learn a maximum of linguistic events (n-grams, part of speech, etc.) [9]. In other words statistical processing of corpora allows to get knowledge by studying recurrent phenomenon. A corpus should be large in order to model statistically a maximum of reliable constructions. The more a corpus is important, the better the events are modeled [9]. For machine translation or speech recognition, it is not surprising to train the language model on a corpus of more than 300 million of words. Classical n-grams models are often enriched by language models based on triggers which are used in several domains, for example in translation, they are exploited to build multilingual dictionaries [11].

We use the triggers to enrich our ontology, our aim is to find terms that are semantically related to the terms of the ontology, then we integrate them into the ontology, to better represent its concepts. The triggers focus on terms that often appear together. That is to say, a term $w_{i}$ will probably trigger the term $w_{j}$. That means we can predict the term $w_{j}$ when $w_{i}$ occurs (it can be written as: $w_{i} \rightarrow w_{j}$ ). For instance the term plane will probably predict the term travel. The triggers are determined by calculating for each ontology term its Mutual Information with each term in the dictionary. Then, only terms with a high mutual information are kept and are used as triggered terms [9]. The mutual information is a measure of distance stemming from the information theory, which allows to measure the degree of association between two events. The mutual information $M I(x, y)$ represents the importance of the relationship between two events $x$ and $y$. The non-weighted $M I$ is given below:

$$
M I(x, y)=\log \frac{P(x, y)}{P(x) P(y)}
$$

where:

$P(x)$ is the marginal probability of $\mathrm{x}$

$P(y)$ is the marginal probability of $\mathrm{y}$

$P(x, y)$ is joint probability xy

In the formula (1) the event $x$ represents the term trigger, $y$ represents its triggered term in the corpus and $x y$ illustrates the occurrence of trigger with its triggered term in the corpus.

We use this principle of trigger to enrich ontology at the level of concepts, i.e. each triggered term will be integrated as synonym, as form or as reason in concept by hand. This model allowed to enrich "sad" concept with terms: "tear", "painful", "hurt" and "annoyed", as shown in figure 2.

In the next section, we present the aim of conceptualizing of the conflicts domain. The concepts of the ontology will be used to detect the conflicts emails. 


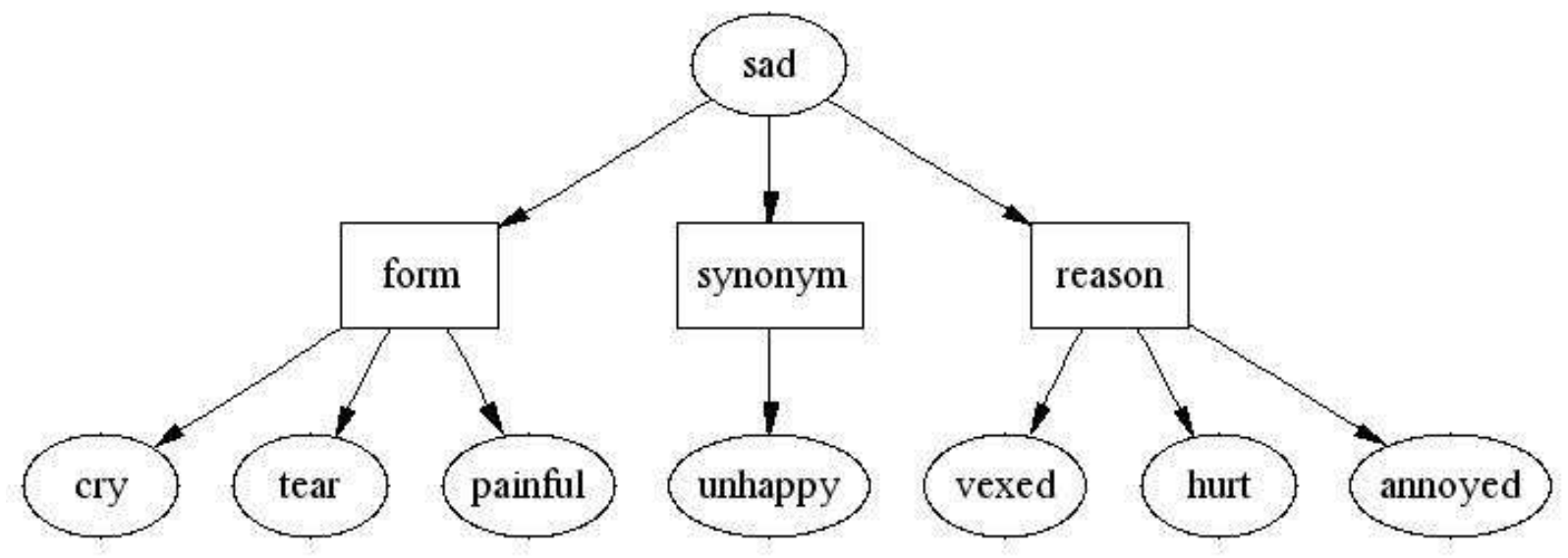

Fig. 2. The sad emotion after the addition of the triggers

\section{Classification emails}

Our approach solves the task of detecting conflicts in the emails by their classification, it consists to identify the concept to which an email belongs to and therefore to recognize the emotion expressed in this email. The domain of classification is made up of two distinct approaches: supervised and unsupervised learning. The distinction between these two approaches comes from the knowledge or not of categories. Indeed, supervised classification learns to assign instances to predefined categories, but unsupervised classification is a task, which learns classification from the data, because categories are unknown. For the purposes of this paper we will focus on supervised learning. We classify emails according to concepts of ontology, i.e. that the categories of classification are emotions of ontology.

\subsection{The classification model}

Each email $\left(M_{i}\right)$ to classify is coded by a vector according to the terms of a concept $\left(C_{i}\right)$. Then a similarity is calculated to quantify the semantic proximity between the email (its representation by the concept vector) and an emotion. This process is repeated for each emotion. Once all similarities are calculated, the classification process associates to each email the emotion with the highest similarity.

$$
C_{i}=\left\{c_{i 1} \ldots, c_{i j}, \ldots, c_{i n}\right\}
$$

where $c_{i j}$ is the weight of the term $w_{j}$ in the ith concept, and $n$ is the number of terms in the concept which varies from one concept to another.

$$
M_{k}=\left\{m_{i 1}, \ldots, m_{i j}, \ldots, m_{i n}\right\}
$$


where $m_{i j}$ is the weight of the term $w_{j}$ in the ith concept. Weights are estimated using the TF*IDF (Term Frequency, Inverse Document Frequency):

$$
\begin{gathered}
m_{i j}=T F\left(w_{i j}, M\right) \times I D F\left(w_{i j}\right) \\
I D F\left(w_{i j}\right)=\log \left(\frac{T}{t_{j}}\right)
\end{gathered}
$$

where $T F\left(w_{i j}, M\right)$ is the frequency of the term $w_{i j}$ of the ith concept within the email $M . T$ is the size of the corpus of the $i t h$ concept and $t_{j}$ is the number of emails in which the term $w_{i j}$ occurs.

The classification is done by calculating for each pair $\left(C_{i}, m_{i}\right)$ the cosine of the angle between vectors $C_{i}$ and $m_{i}$ defined as follows:

$$
\operatorname{Cos}\left(C_{i}, M_{i}\right)=\frac{\sum_{j=1}^{n} c_{i j} m_{i j}}{\sqrt{\sum_{j=1}^{n} c_{i j}{ }^{2} \sum_{j=1}^{n} m_{i j}^{2}}}
$$

\subsection{Labeling}

We labelled the corpus according to the emotions of our ontology. We set up a semiautomatic procedure to label the corpus, first we automatically labeled the corpus through a function that we developed, then we manually corrected the errors of the function.

\section{Evaluation and Proposals}

To evaluate our email classification model, we have chosen to use precision, recall and F measure. Recall is defined as the fraction of relevant emails that are retrieved by the system; and Precision is defined as the fraction of retrieved emails that are in fact relevant. The F measure characterizes the combined performance of Recall and Precision. These measures are calculated as follows [9]:

$$
\begin{gathered}
\text { Recall }=\frac{\text { Number of relevant emails retrieved }}{\text { Number of emails to retrieve }} \\
\text { Precision }=\frac{\text { Number of relevant emails retrieved }}{\text { Number of emails retrieved }} \\
\text { Fmeasure }=2 \times \frac{\text { Precision } \times \text { Recall }}{\text { Precision }+ \text { Recall }}
\end{gathered}
$$

Recall and precision are often used because they reflect the point of view of the user: if precision is low, the team leader will be dissatisfied, because he will waste time for reading emails which do not deal with conflicts, and if recall is low, he will not access to the emails of conflicts. 
We used two other measures to estimate the performance of a system from its errors, namely the False Acceptance (FA), where an email is wrongly considered as conflictual, and the False Rejection (FR), where an email is wrongly rejected. These measures are calculated as follows [9]:

$$
\begin{aligned}
& F A=\frac{\text { Number of False Acceptances }}{\text { Number of emails retrieved }} \\
& F R=\frac{\text { Number of False Rejections }}{\text { Number of emails to retrieve }}
\end{aligned}
$$

For our experiments, we used a corpus of "Le Monde" newspaper, it is made up of 7854 paragraphs, we have divided into two sets, $90 \%$ of the corpus for training and the $10 \%$ remaining for test. We first evaluated our classifier based on the use of the first version of the ontology (table 1). Then we evaluated it again when we use the version of ontology augmented by triggers (table 2). The experiments given below illustrates results on only few concepts of our ontology. These results show that triggers have allow to maximize classification performance, for certain concepts all measures have increased, as "treason", for others, precision and false acceptance show that the triggers have improved the accuracy of the selection of conflicts emails, such as "fear".

Table 1. Performance of the classifier with the initial ontology

\begin{tabular}{|l|l|l|l|l|l|}
\hline Concept & Recall & Precision & F-Measure & FA & FR \\
\hline fear & 0.98 & 0.91 & 0.94 & 0.091 & 0.020 \\
\hline disgust & 0.96 & 0.93 & 0.94 & 0.069 & 0.036 \\
\hline treason & 0.84 & 0.84 & 0.84 & 0.158 & 0.158 \\
\hline arrogance & 1.0 & 0.75 & 0.86 & 0.25 & 0.0 \\
\hline protest & 0.81 & 1.0 & 0.9 & 0.0 & 0.186 \\
\hline discouragement & 1.0 & 0.77 & 0.87 & 0.225 & 0.0 \\
\hline \hline Average on all the concepts & 0.93 & 0.87 & 0.89 & 0.132 & 0.067 \\
\hline
\end{tabular}

Table 2. Performance of the classifier by using the augmented ontology

\begin{tabular}{|l|l|l|l|l|l|}
\hline Concept & Recall & Precision & F-Measure & FA & FR \\
\hline fear & 0.96 & 0.97 & 0.96 & 0.031 & 0.041 \\
\hline disgust & 1.0 & 0.9 & 0.95 & 0.103 & 0.0 \\
\hline treason & 0.94 & 0.92 & 0.93 & 0.075 & 0.057 \\
\hline arrogance & 0.9 & 0.99 & 0.94 & 0.011 & 0.103 \\
\hline protest & 0.89 & 1.0 & 0.94 & 0.0 & 0.105 \\
\hline discouragement & 0.96 & 0.9 & 0.93 & 0.103 & 0.037 \\
\hline \hline Average on all the concepts & 0.94 & 0.95 & 0.94 & 0.054 & 0.057 \\
\hline
\end{tabular}




\section{Discussion and conclusion}

The classifier achieve good performance in terms of recall and precision. Increasing the ontology by triggers allows us to outperfom the results obtained by the initial ontology. In fact, the initial ontology has been developped by hand. For each concept only few words have been used. Using triggers allows to adapt the ontology to the used corpus. Nowadays, we concentrate our effort in collecting more appropriate and significant corpus from certain forums in which conflicts are more frequent than what we get from our "Le Monde" corpus, in order to test our ontology in its context, because we have found a cases of polysemy. However, it is necessary to consider the problem of polysemy in the detection of emails of conflict. We propose to take advantage of the relations between emotions. An email which includes the expression of two emotions or more, it has more chances to represent a conflict between two persons, for instance the existence of concept "anger" with concept "humiliation".

\section{References}

1. R. S. Broches. Unraveling the Hawthorne Effect: An Experimental Artifact 'Too Good to Die'. PhD thesis, University of Wesleyan, April 2008.

2. C. Cortes and V. Vapnik. Support vector networks. In Machine Learning, pages 273-297, 1995.

3. N. Debois. Les émotions en eps : comprendre et intervenir. EP.S, 1:7, 2007.

4. E. Denoual. Méthodes en caractères pour le traitement automatique des langues. $\mathrm{PhD}$ thesis, University of Joseph Fourier, 2006.

5. G. Dias. Extraction automatique d'associations lexicales à partir de corpora. $\mathrm{PhD}$ thesis, University of Orleans, December 2002.

6. C. Enguehard and L. Pantera. Automatic natural acquisition of a terminology. Journal of Quantitative Linguistics, 2(1):27-32, 1995.

7. N. Friedman, D. Geiger, and M. Goldszmidt. Bayesian network classifiers. Mach. Learn., 29(2-3):131-163, 1997.

8. T. Gruber. A translation approach to portable ontology specifications. Knowledge Acquisition, 5(2):199-220, 1993

9. J. Haton, C. Cerisara, D. Fohr, Y. Laprie, and K. Smaili. Reconnaissance automatique de la parole. Du signal à son interprétation. Dunod, 2006.

10. M. Larivery. Les genres d'émotions. La lettre du psy, 2(7), July 1998.

11. C. Lavecchia, K. Smaïli, D. Langlois, and J.-P. Haton. Using inter-lingual triggers for machine translation. In Eighth conference INTERSPEECH, 2007.

12. D. L. McGuinness. Ontologies come of age. In D. Fensel, J. Hendler, H. Lieberman, and W. Wahlster, editors, Spinning the Semantic Web: Bringing the World Wide Web to Its Full Potential, pages 171-194. MIT Press, 2002.

13. G. Salton, A. Wong, and C. S. Yang. A vector space model for automatic indexing. Commun. ACM, 18(11):613-620, 1975.

14. R. Voltz, D. Oberle, S. Staab, and B. Motik. Kaon server - a semantic web management system. In Alternate Track Proceedings of the Twelfth International World Wide Web Conference, pages 139-148, Budapest, Hungary, May 2003. ACM.

15. S. Whittaker and C. Sidner. Email overload: exploring personal information management of email. In CHI '96: Proceedings of the SIGCHI conference on Human factors in computing systems, pages 276-283, New York, NY, USA, 1996. ACM Press. 